BULL. AUSTRAL. MATH. SOC.

\title{
IDEALS GENERATED BY SINGULAR INNER FUNCTIONS
}

\author{
Michael von Renteln
}

\begin{abstract}
Singular inner functions are in many respects the most important and difficult type of functions in the Banach algebra $H^{\infty}$ of bounded analytic functions in the unit disc. This paper is concerned with ideals generated by singular inner functions. In particular, conditions on the associated measures are given so that the ideal spans the whole algebra $H^{\infty}$. To this end the local boundary behavior of a singular inner function is studied and the results obtained there may be of independent interest.
\end{abstract}

\section{Introduction}

Let $D$ denote the open unit disc in the complex plane, let $\bar{D}$ denote its closure, and let $T$ denote its boundary, the unit circle. $H^{\infty}$ is the Banach algebra of all bounded analytic functions in $D$ under the usual pointwise operations and the sup-norm . $\left(f_{1}, \ldots, f_{N}\right)$ denotes the ideal generated by the functions $f_{1}, \ldots, f_{N} \in H^{\infty}$. Probably the most well known theorem about the ideal structure of $H^{\infty}$ is the Corona Theorem. One version goes as follows.

CORONA THEOREM. Let $f_{1}, \ldots, f_{N}$ be a finite number of functions in $H^{\infty}$ with

$$
\inf \left\{\sum_{i=1}^{N}\left|f_{i}(z)\right|: z \in D\right\}>0,
$$

then it folzows that $\left(f_{1}, \ldots, f_{N}\right)=H^{\infty}$.

Received 28 September 1979. 
Every function $f \in H^{\infty}, f \neq 0$, has the following canonical factorization

$$
f=B \cdot S_{\mu} \cdot F,
$$

where $B$ is a Blaschke product, $S_{\mu}$ is a singular inner function and $F$ is an outer function. For details the reader is referred to Hoffman [2]. A singular inner function is a function of the form

$$
S_{\mu}(z):=\exp \left[-\int_{T} \frac{e^{i t}+z}{e^{i t}-z} d \mu\right],
$$

where $\mu$ is a finite positive Borel measure on $T$, singular with respect to Lebesgue measure $m$ on $T$.

The modulus of $S_{\mu}$ is

$$
\begin{gathered}
\left|S_{\mu}(z)\right|=\exp \left[-\int_{T} P(z, t) d \mu\right] . \\
P(z, t)=\operatorname{Re}\left(\frac{e^{i t}+z}{e^{i t}-z}\right)=\frac{1-r^{2}}{\left|z-e^{i t}\right|^{2}}=P_{p}(\theta-t)
\end{gathered}
$$

denotes the Poisson kernel, where $z=r e^{i \Theta}, 0 \leq r<1 .-\pi \leq \theta<\pi$.

The purpose of this paper is to discuss ideals in $H^{\infty}$ which are generated by singular inner functions $S_{i}=S_{\mu_{i}}, 1 \leq i \leq N$, with associated singular measures $\mu_{i}$. We are especially interested in the following problem.

PROBLEM. What conditions on the singular measures $\mu_{1}, \ldots, \mu_{N}$ ensure that the associated singuzar irmer functions generate the whole algebra $H^{\infty}$, that is $\left(S_{1}, \ldots, S_{N}\right)=H^{\infty}$ ?

To attack this problem we provide in Section 2 the measure theoretic preliminaries. In Section 3 we give a necessary and also a sufficient condition for $\left(S_{1}, \ldots, S_{N}\right)=H^{\infty}$. The conditions depend only on the distributions of the measures $\mu_{i}$ on $T, I \leq i \leq N$. For an appropriate study of the problem one has also to consider the concentrations of the measures. Conditions involving the concentrations are given in Section 4 . 
In Section 5 necessary and sufficient conditions for certain classes of generators are obtained.

Let us say one word about earlier considerations of singular inner functions in the literature and the difference of our investigations. In many areas of function theory and functional analysis singular inner functions play an important rôle, for example in the theory of the Hardy spaces $H^{p}$, especially in those where $0<p<1$ (see, for example, [6]). Investigations in those areas deal with the question of how global properties of the singular inner function $S_{\mu}$ are affected by global properties of the measure $\mu$; for example how the smoothness of the measure $\mu$ (measured in terms of the modulus of continuity) influences the growth of the maximum or the mean modulus of the corresponding singular inner function $S_{\mu}$ (see [6], p. 114 f. and p. 119).

Our considerations of the singular measures are quite different, since for our problems in algebraic ideal theory only the local behavior of the singular measures are important. We especially need estimates of the modulus of positive harmonic functions inside specific oricycles tangent to the unit circle. These results may be of independent interest.

\section{Measure theoretic preliminaries}

All measures we consider are finite positive Borel measures on the unit circle $T$. A singular measure is a measure singular with respect to Lebesgue measure $m$ on $T$. By $\operatorname{supp}(\mu)$ we denote the (closed) support of the measure $\mu$. We shall say that a measure $\mu$ is concentrated on a Borel set $E \subset T$, if $\mu(T \backslash E)=0$. Then the support of the measure $\mu$ may also be defined as the smallest closed set on which the measure $\mu$ is concentrated. A singular measure $\mu$ is concentrated, by definition, on a set of Lebesgue measure zero, but the support of $\mu$ may be the whole set $T$. $\|\mu\|$ denotes the total variation norm of $\mu$. Let us start with some notation.

NOTATION. Let $a=e^{i \alpha} \in T$. Then

$\delta_{a}$ is the Dirac measure (point mass) associated with the point $a$; 
$J(\alpha, s):=\left\{e^{i t}: \alpha-s<t<\alpha+s\right\}$ is an open circular arc of length $2 s$ with center $a$, where $0<s \leq \pi$.

LEMMA 2.1. Suppose $\mu$ is a singular measure. Then $\mu$ is concentrated on the set

$$
U(\mu):=\left\{\alpha \in T:(D \mu)(a):=\lim _{s \rightarrow 0}(\mu / m)[J(a, s)]=\infty\right\} .
$$

Proof. For short we write $U$ instead of $U(\mu)$. Since $\mu$ is positive and singular it follows from the Decomposition Theorem of de la Vallée Poussin (see, for example, Saks [5], p. 127, 9.7) that $\mu(E)=\mu(E \cap U)$ for every Borel set $E \subset T$. Hence we obtain $\mu(T)=\mu(U)$ and $\mu(T \backslash U)=\mu(T)-\mu(U)=0$.

REMARKS. I. $(D \mu)(a)$ is called the symmetric derivative of the measure $\mu$ at the point $a$.

2. Note that from Theorem 14.5 of Saks ([5], p. 151) follows only the weaker result: $\mu$ is concentrated on the set where the upper symmetric derivative $\bar{D} \mu$ is infinite.

3. A proof without using the Decomposition Theorem of de la Vallée Poussin may be patterned after the proof of Theorem 8.9 of Rudin ([3], p. 158). Note that $D \mu$ there is not the symmetric derivative of $\mu$. The symmetric derivative may exist at points at which the derivative of Theorem 8.9 does not exist. Therefore Theorem 8.9 implies Lemna 2.1.

4. Every singular measure $\mu$ has a decomposition $\mu=\sigma+\nu$, where $\sigma$ is a discrete singular measure and $\nu$ is a continuous singular measure. $\sigma$ is concentrated on the set where $\mu$ is discontinuous. This is a countable set and also the smallest set where $\sigma$ is concentrated. This set is in general much smaller than $U(\sigma)$ (see Remark 5). On the other hand there is no smallest set where $\nu$ is concentrated. $\nu$ continuous at $a \in T$ means $\nu(\{a\})=0$. This implies $\nu(A)=0$ for each countable set $A$ of $T$. If $\nu$ is concentrated on a set $M$, then $v$ is also concentrated on $M \backslash A$, where $A$ is countable.

5. If $U(\mu)$ is countable, then $\mu$ is a discrete singular measure. The converse statement is false. For any given closed set $E \subset T$ of zero Lebesgue measure we can construct a discrete singular measure $\sigma$ with $U(\sigma)=E$. 
LEMMA 2.2. Suppose $\mu$ is a singular measure. Let

$$
L(\mu):=\left\{a \in T: \lim _{r \rightarrow 1} S_{\mu}(r a)=0\right\} .
$$

Then it follows

$$
U(\mu) \subset L(\mu) \subset \operatorname{supp}(\mu)=\overline{U(\mu)} .
$$

Proof. The first inclusion follows from Fatou's Theorem ([3], Theorem 11.10, p. 226): if the symetric derivative $\left(D_{\mu}\right)(a)$, finite or infinite, exists at a point $a \in T$, then we have

$$
\lim _{r \rightarrow 1}\left|S_{\mu}(r a)\right|=\exp [-2 \pi D \mu(a)] \text {. }
$$

The inclusion $L(\mu) \subset \overline{U(\mu)}$ follows from Lemma 2.1 and Fatou's Theorem. $\operatorname{supp}(\mu)=\overline{U(\mu)}$ is clear from the definition of $\operatorname{supp}(\mu)$.

REMARK. Rudin has given a nice example of a singular measure $\mu$ ([4], p. 381 f.) which shows that in general $U(\mu)$ is a proper subset of $L(\mu)$. He considers the half-space in $\mathbb{R}^{n}$, but his example works also in the unit disc. The measure $\mu$ has the form

$$
\mu=\sum_{n=1}^{\infty} s_{n} \delta\left(a_{n}\right),
$$

with, for example, $s_{n}=1 / n$ ! and $\arg \left(a_{n}\right)=\sqrt[3]{n} /(n+1) !$. A calculation shows for the lower symmetric derivative $(D \mu)(1)=0$, and for the upper symmetric derivative $(\bar{D} \mu)(1)=\infty$, but $\lim _{r \rightarrow 1} S_{\mu}(r)=0$.

\section{Conditions depending on the distributions of the generator-measures}

In this section we will give a necessary and also a sufficient condition for $\left(S_{1}, \ldots, S_{N}\right)=H^{\infty}$ which depends only on the sets where the associated singular measures $\mu_{1}, \ldots, \mu_{N}$ are concentrated.

THEOREM 3.1. In order that the ideal generated by the singular imer functions $S_{1}, \ldots, S_{N}$ is the whole algebra $H^{\infty}$ the condition 


$$
\bigcap_{i=1}^{N} U\left(\mu_{i}\right)=\varnothing
$$

is necessary, and the condition

$$
\prod_{i=1}^{N} \overline{U\left(\mu_{i}\right)}=\varnothing
$$

is sufficient.

Proof. The necessity follows from Lemma 2.2. To prove the sufficiency let $M\left(H^{\infty}\right)$ denote the maximal ideal space of $H^{\infty}$. We identify the maximal ideals $M$ of $H^{\infty}$ with the nontrivial multiplicatively linear functionals $m$ on $H^{\infty}$. Define a map

$$
\pi: M\left(H^{\infty}\right) \rightarrow \bar{D} \text { by } \pi: m \rightarrow m(z)
$$

where $z$ is the identity function. For a point $a=e^{i \alpha} \in T$ let $M_{a}:=\pi^{-1}(a)$ be the fiber over $a$.

Assume $\left(S_{1}, \ldots, S_{N}\right) \neq H^{\infty}$, then there exists a fiber $M_{a}$ and a maximal ideal $M \in M_{a}$ with $s_{1}, \ldots, s_{N} \in M$. By a result of schark (see Hoffman [2], pp. 161, 162) we have for every $i \in\{1, \ldots, N\}$ :

$$
\lim _{z \rightarrow a} \inf \left|S_{i}(z)\right|=0 \text {. }
$$

From our hypothesis it follows that there is an index $j \in\{1, \ldots, N\}$, such that $a \notin \overline{U\left(\mu_{j}\right)}$. Then

$$
\delta:=\frac{1}{2} \operatorname{dist}\left[a, \overline{U\left(\mu_{j}\right.}\right]>0 .
$$

For $z=r e^{i \theta}$ with $0 \leq r<1,|\Theta-\alpha|<\delta$, we have

$$
\int_{T} P(z, t) d \mu_{j}=\int_{|t-\alpha| \geq 2 \delta} P(z, t) d \mu_{j} \leq \int_{|t-\alpha| \geq 2 \delta} \frac{d \mu_{j}}{\left|e^{i t}-z\right|^{2}} \leq \frac{\left\|\mu_{j}\right\|}{\sin ^{2} \delta} .
$$

This implies $\left|S_{j}(z)\right| \geq \varepsilon$ for some $\varepsilon>0$, which is a contradiction to (3.1).

COROLLARY 3.2. Suppose that the sets $U\left(\mu_{i}\right), 1 \leq i \leq N$, are 
closed. Then $\left(S_{1}, \ldots, S_{N}\right)=H^{\infty}$, if and only if $\bigcap_{i=1}^{N} U\left(\mu_{i}\right)=\emptyset$.

REMARK. The second condition in Theorem 3.1 is also necessary in the case $N=1$, but not necessary for $N \geq 2$, as follows from Theorem 4.4 .

\section{Conditions depending on the concentrations of the generator-measures}

To study the problem further we have to relate the behavior of $\left|S_{\mu}(z)\right|$ near a boundary point $a \in T$ to the behavior of the measure $\mu$ in a neighbourhood of that point. It turns out that some special tangential approaches $z \rightarrow a$ are essential for our considerations. Therefore we shall begin by introducing some notation.

NOTATION. Let $a \in T, 0<R<\frac{1}{2}$, and $\mu$ be a positive Borel measure on $T$. Then

$$
K(a, R):=\{z:|z-(1-R) a|<R\}
$$

is the interior of the oricycle of radius $R$ internally tangent to $T$ at the point $a$, especially we put

$$
\begin{gathered}
K(a):=K\left(a, \frac{1}{2}\right) . \\
G(\mu):=U\{K(a): a \in U(\mu)\}
\end{gathered}
$$

is the union of the oricycles $K(a)$ at those points $a \in T$, where $\mu$ has an infinite symmetric derivative.

Before going to Lemma 4.1 we study a special example.

EXAMPLE. Let $\mu=\delta_{1}$ be the Dirac measure associated to the point

1. Then

$$
\begin{aligned}
S_{\mu}(z) & =\exp [-(1+z) /(1-z)], \\
U(\mu) & =L(\mu)=\operatorname{supp}(\mu)=\{1\}
\end{aligned}
$$

and

$$
\left|S_{\mu}(z)\right|=1 / e=S_{\mu}(0)
$$

holds for all $z \neq 1$ on the oricycle $\left\{z:\left|z-\frac{1}{2}\right|=\frac{1}{2}\right\}$. Thus $\left|S_{\mu}(z)\right| \geq S_{\mu}(0)$ for all $z \in D \backslash G(\mu)$. 
It is perhaps surprising that the last inequality in the example holds for any singular inner function. This shows the following lema.

LEMMA 4.1. Let $S_{\mu}$ be a singular irner function. Then $\left|S_{\mu}(z)\right| \geq S_{\mu}(0)$ holds for all $z \in D \backslash G(\mu)$.

The positive constant $S_{\mu}(0)$ is best possible.

Proof. Let $z \in r e^{i \theta} \in D \backslash G(\mu)$. Then for every $e^{i t} \in U(\mu)$ it follows $\left|z-\frac{1}{2} e^{i t}\right| \geq \frac{2}{2}$. Thus

$$
1-r \leq 1-\cos |\theta-t|=2 \sin ^{2}((|\theta-t|) / 2) \text {. }
$$

This implies

$$
\begin{aligned}
\int_{T} P(z, t) d \mu & =\int_{U(\mu)} \frac{1-r^{2}}{(1-r)^{2}+4 r \sin ^{2}((|\theta-t|) / 2)} d \mu \\
& \leq \int_{U(\mu)} \frac{1-r^{2}}{(1-r)^{2}+2 r(1-r)} d \mu=\int_{T} d \mu=\|\mu\| .
\end{aligned}
$$

Thus $\left|S_{\mu}(z)\right| \geq \exp (-\|\mu\|)=S_{\mu}(0)$.

Since $z=0 \in D \backslash G(\mu)$ for every measure $\mu$, the constant $S_{\mu}(0)$ is best possible.

REMARK. In the proof of Lemma 4 . I we have not used that $\mu$ is a singular measure. But if $\mu$ is not singular, then the set $D \backslash G(\mu)$ in general consists only of the point 0 and in that case the statement is trivial.

DEFINITION 4.2. Let $a=e^{i \alpha}$ be a point of $T$ and $\mu$ be a finite positive Borel measure on $T$. Then we define:

$$
\begin{aligned}
M(\mu, a, \theta) & :=\sup \left\{(\mu / m)\left[J\left(e^{i \theta}, s\right)\right]:((2 / \pi)|\theta-\alpha|)^{2}<s \leq \pi\right\} ; \\
N(\mu, \alpha) & :=\sup \{M(\mu, a, \theta):|\theta-\alpha|<\pi / 2\} .
\end{aligned}
$$

REMARKS. 1. In the special case $\theta=\alpha$ and $\mu$ absolutely continuous $M(\mu, a, \theta)$ is the Hardy-Littlewood maximal function.

2. If the measure $\mu$ is discontinuous at the point $a$, that is $\mu(\{a\})>0$, then it follows $N(\mu, a)=\infty$. On the other hand $N(\mu, a)=\infty$ 
does not imply $\mu(\{a\})>0$.

LEMMA 4.3. Suppose $S_{\mu}$ is a singular inner function and $a \in T$. Then it follows

$$
\left|S_{\mu}(z)\right| \geq \exp [-C N(\mu, a)]
$$

for all $z \in K(a)$, where $C$ is an absolute constant.

Proof. Fix a point $z=r e^{i \theta} \in K(a)$. For convenience, we shall assume $a=1$. According to the point $z$ we decompose the unit circle $T$ in a union of sets $T_{n}$ in a special way. Let $T_{n}=T_{n}(r, \theta)$ be defined as follows:

$$
\begin{aligned}
& T_{0}:=\left\{e^{i t}:|t-\theta| \leq 1-r\right\}, \\
& T_{n}:=\left\{e^{i t}: 2^{n-1}(1-r)<|t-\theta| \leq 2^{n}(1-r)\right\}, n \in \mathbb{N} .
\end{aligned}
$$

For $n=0,1,2, \ldots$ let $I_{n}:=\bigcup_{k=0}^{n} T_{k}$. Then

$$
m\left(T_{n}\right) \leq m\left(I_{n}\right)=2^{n+1}(1-r)
$$

Let $p$ be the smallest integer $n$ with $I_{n} \supset T$. We put $I_{p}=T$.

By standard estimations of the Poisson kernel there is an absolute constant $M$, such that

$$
\begin{aligned}
\int_{T} P(z, t) d \mu & \leq M \int_{T} \frac{(1-r)}{(1-r)^{2}+(\theta-t)^{2}} d \mu \\
& \leq M\left\{\int_{T_{0}} \frac{1-r}{(1-r)^{2}} d \mu+\sum_{n=1}^{p} \int_{T_{n}} \frac{1-r}{(\theta-t)^{2}} d \mu\right\} \\
& \leq M\left\{\frac{1}{1-r} \int_{T_{0}} d \mu+\sum_{n=1}^{p} \frac{1}{2^{2 n-2}(1-r)} \int_{T_{n}} d \mu\right\} \\
& \leq M\left\{2(\mu / m)\left(I_{0}\right)+8 \sum_{n=1}^{p} \frac{1}{2^{n}}(\mu / m)\left(I_{n}\right)\right\} \\
& \leq M\left\{2+8 \sum_{n=1}^{p} \frac{1}{2^{n}}\right\} \cdot \sup \left\{(\mu / m)\left[J\left(e^{i \theta}, s\right)\right]:(1-r) \leq s \leq \pi\right\} \\
& \leq 10 M \cdot M(\mu, a, \theta) \leq 10 M \cdot N(\mu, a) .
\end{aligned}
$$


From this the result follows. We have used that $z \in r e^{i \theta} \in K(1)$ implies $-\pi / 2<\theta<4 / 2$ and $1-r \geq 1-\cos \theta=2 \sin ^{2}(\theta / 2) \geq(2 / \pi)^{2} \theta^{2}$.

THEOREM 4.4. Suppose that the measures $\mu_{1}, \ldots, \mu_{N}$ fulfill the folzowing condition

$$
\min _{1 \leq j \leq N} \sup _{a \in U\left(\mu_{j}\right)} \min _{1 \leq i \leq N} N\left(\mu_{i}, a\right)<\infty .
$$

Then it follows that $\left(S_{1}, \ldots, S_{N}\right)=H^{\infty}$.

Proof. By hypothesis there exists an index $j \in\{l, \ldots, N\}$ and an absolute constant $M$, such that

$$
\min _{1 \leq i \leq N} N\left(\mu_{i}, a\right) \leq M \text { for every } a^{\prime} \in U\left(\mu_{j}\right) \text {. }
$$

Let $k$ be the index (depending on $a$ ) where the minimum is attained. Lemma 4.3 implies

$$
\left|S_{k}(z)\right| \geq \exp [-C M]=: \delta_{1}>0 \quad[z \in K(a)] .
$$

From this inequality we obtain

$$
\sum_{i=1}^{N}\left|s_{i}(z)\right| \geq \delta_{1} \quad\left[z \in G\left(\mu_{j}\right)\right] .
$$

On the other hand, Lerma 4.1 implies

$$
\left|S_{j}(z)\right| \geq \delta_{2}>0 \quad\left[z \in D \backslash G\left(\mu_{j}\right)\right] .
$$

The last two inequalities together with the Corona Theorem yield the desired result.

COROLLARY 4.5. Suppose that there exists an absolute constant $M$ and for every $a=e^{i \alpha} \in U\left(\mu_{1}\right)$ an index $j$ (depending on $a$ ) such that

$$
\mu_{j}[J(a, s)] \leq M m^{2}[J(a, s)]
$$

holds for all $s$ with $0<s \leq \pi$. Then it follows that $\left(s_{1}, \ldots, s_{N}\right)=H^{\infty}$.

Proof. By Theorem 4.4 it is enough to show that condition (4.1) implies $N\left(\mu_{j}, a\right) \leq C$ with an absolute constant $C$. We estimate 


$$
\begin{aligned}
\mu_{j}\left[J\left(e^{i \theta}, s\right)\right] \leq \mu_{j}[J(a,|\Theta-\alpha|+s)] & \leq M m^{2}[J(\alpha,|\theta-\alpha|+s)] \\
& =4 M(|\theta-\alpha|+s)^{2}=4 M\left(|\theta-\alpha|^{2}+2|\theta-\alpha| s+s^{2}\right) .
\end{aligned}
$$

For all $s$ with $((2 / \pi)|\theta-\alpha|)^{2}<s \leq \pi$ and all $\theta$ with $|\Theta-\alpha|<\pi / 2$ we get

$$
\mu_{j}\left[J\left(e^{i \theta}, s\right)\right] \leq M \pi^{2}(1+|\theta-\alpha|+s) s
$$

that is,

$$
\mu_{j}\left[J\left(e^{i \theta}, s\right)\right] \leq C m\left[J\left(e^{i \theta}, s\right)\right]
$$

with some other absolute constant $C$. We conclude that $N\left(\mu_{j}, a\right) \leq C$.

REMARK. One can show that the exponent 2 in condition (4.1) cannot be replaced by any exponent smaller than 2 .

COROLLARY 4.6. Suppose that for every $a=e^{i \alpha} \in U\left(\mu_{1}\right)$ there exists a generator $S_{j}$ such that $S_{j}$ has a finite angular derivative at $a$, that is $\left|S_{j}^{\prime}(a)\right| \leq M$, where $M$ is independent of $j$ and $a$. Then it folzows that $\left(S_{1}, \ldots, S_{N}\right)=H^{\infty}$.

Proof. From a theorem of M. Riesz (see [1], p. 117 for a short proof) it follows that

$$
\left|S_{j}^{\prime}(a)\right|=2 \int_{T} \frac{d \mu}{j} \frac{\left.j\right|^{i t}-\left.a\right|^{2}}{\mid e^{2}} .
$$

We show that $\mu_{j}$ fulfills condition (4.1).

$$
\begin{aligned}
\mu_{j}[J(a, s)]=\int_{\alpha-s}^{\alpha+s} d \mu_{j} \leq s^{2} \int_{\alpha-s}^{\alpha+s} \frac{d \mu_{j}}{|t-\alpha|^{2}} & \\
& \leq m^{2}[J(a, s)] \int_{T} \frac{d \mu_{j}}{\left|e^{i t}-a\right|^{2}} \leq M m^{2}[J(a, s)] .
\end{aligned}
$$

\section{Necessary and sufficient conditions}

In this section we shall show that the condition of Theorem 4.4 is also necessary for a large class of generators. Before turning to this 
question we need the following two lemmas.

LEMMA 5.1. A singular inner function $S_{\mu}$ is bounded away from zero in $K(a), a \in T$, if and only if $N(\mu, a)<\infty$.

Proof. One direction follows from Lemma 4.3. To prove the converse, assume that $S_{\mu}$ is bounded away from zero in $K(a)$. Then there exists an absolute constant $M$, such that for every $z \in K(a)$ we have

$$
\int_{T} P(z, t) d \mu \leq M .
$$

Without loss of generality, we may take $a=1$. Let $\theta \in(-\pi / 2,+\pi / 2)$ and $\frac{z}{2}>s>((2 / \pi) \theta)^{2}$ be given. We choose the point $z=r_{s} e^{i \theta}$ with $r_{s}=1-2 s$. Since $r_{s} \leq 1-2((2 / \pi) \theta)^{2}<\cos \theta$ we see that $z$ is in $K(1)$. Applying the inequality (5.1) for our specific point $z$ we get

$$
\begin{aligned}
& M \geq \int_{T} P(z,t) d \mu \geq \int_{T} \frac{1-r_{s}}{\left(1-r_{s}\right)^{2}+(\theta-t)^{2}} d \mu \\
& \quad=\int_{T} \frac{2 s}{4 s^{2}+(\theta-t)^{2}} d \mu \geq 2 / 5 s \int_{|\theta-t|<s} d \mu=(4 / 5)(\mu / m)\left[J\left(e^{i \theta}, s\right)\right] .
\end{aligned}
$$

Since $M$ is independent of $\theta$ and $s$ we obtain

$$
\sup \left\{(\mu / m)\left[J\left(e^{i \theta}, s\right)\right]:((2 / \pi) \theta)^{2}<s<\frac{1}{2}\right\} \leq M
$$

for all $\theta \in(-\pi / 2,+\pi / 2)$. This implies $N(\mu, a)<\infty$.

LEMMA 5.2. If a singular inner function $S_{\mu}$ is bounded caway from zero inside some oricycle $K(\alpha, R), a \in T, 0<R<\frac{3}{2}$, then $S_{\mu}$ is bounded coway from zero in $K(a)$.

Proof. For convenience, assume $a=1$. By hypothesis, there exist $\delta>0$ and $R$ in $\left(0, \frac{2}{2}\right)$ such that

$$
\left|S_{\mu}(z)\right| \geq \delta \text { holds for all } z \in K(1, R) \text {. }
$$

Suppose $S_{\mu}$ is not bounded away from zero in $K(1)$. Then there exists a sequence 


$$
z_{n}=r_{n} e^{i \theta_{n}} \in K(1) \backslash K(1, R), n \in \mathbb{N},
$$

with $z_{n} \rightarrow 1$ and $S_{\mu}\left(z_{n}\right) \rightarrow 0$ for $n \rightarrow \infty$; that is,

$$
\int_{T} P\left(z_{n}, t\right) d \mu \rightarrow \infty \quad(n \rightarrow \infty) .
$$

From (5.3) it follows that, for all $n \geq n_{0}(R)$,

$$
c_{1} \theta_{n}^{2} \leq 1-r_{n} \leq c_{2} \theta_{n}^{2}
$$

with two positive constants $C_{1}$ and $C_{2}, C_{2}$ depending on $R$. For $n \in \mathbb{N}$ let us define $\zeta_{n}=\rho_{n} e^{i \theta_{n}}$ where

$$
1-\rho_{n}=c \Theta_{n}^{2}
$$

and $C>C_{2}$ is a constant (depending on $R$ ) such that $\zeta_{n} \in K(1, R$ ) for all $n \geq n_{1}(R)$. Then

$$
1-r_{n} \leq 1-\rho_{n} \text {. }
$$

We define

$$
M_{n}:=\max _{e^{i t} \in T}\left|\frac{\zeta_{n}-e^{i t}}{z_{n}-e^{i t}}\right| .
$$

Geometric considerations make clear that the maximum is attained for $t=\theta_{n}$ for all $n \in \mathbb{N}$. Thus with (5.5) and (5.6) we obtain

$$
M_{n}=\frac{1-\rho_{n}}{1-r_{n}} \leq \frac{c_{n}^{2}}{C_{1} \Theta_{n}^{2}}=\frac{C}{C_{1}}=: M .
$$

Therefore for all $n \geq n_{3}(R)$ and all $e^{i t} \in T$ we get

$$
\left|\zeta_{n}-e^{i t}\right| \leq M\left|z_{n}-e^{i t}\right|
$$

or 


$$
\frac{1}{M^{2}} \frac{1}{\left|z_{n}-e^{i t}\right|^{2}} \leq \frac{1}{\left|\zeta_{n}-e^{i t}\right|^{2}}
$$

(5.7) and (5.8) now imply

$$
\int_{T} P\left(z_{n}, t\right) d \mu \leq K \int_{T} P\left(\zeta_{n}, t\right) d \mu,
$$

where $K$ is a constant (depending on $R$, but independent of $n$ ). From (5.4) and (5.9) it follows $\left|S_{\mu}\left(\zeta_{n}\right)\right| \rightarrow 0, n \rightarrow \infty$, and this is a contradiction to $(5.2)$.

THEOREM 5.3. Suppose all but one of the generator measures are discrete and let one of the discrete measures be finite. Then $\left(S_{1}, \ldots, S_{N}\right)=H^{\infty}$ if and only if

$$
\min _{1 \leq j \leq N} \sup _{a \in U\left(\mu_{j}\right)} \min _{1 \leq i \leq N} N\left(\mu_{i}, a\right)<\infty .
$$

Proof. The sufficiency follows from Theorem 4.4. To prove the necessity assume without loss of generality that $\mu_{1}, \ldots, \mu_{N-1}$ are discrete measures and $U\left(\mu_{1}\right)$ is finite. We define

$$
V:=\left\{a \in U\left(\mu_{1}\right): \min _{2 \leq i \leq N-1} N\left(\mu_{i}, a\right)<\infty\right\}
$$

Since $U\left(\mu_{1}\right)$ is finite we have

$$
\sup _{a \in V} \min _{2 \leq i \leq N-1} N\left(\mu_{i}, a\right)<\infty
$$

We shall show now that for each $a \in U\left(\mu_{1}\right) \backslash V$ there exists a radius $R \in\left(0, \frac{1}{2}\right)$ such that

$$
\inf \left\{\left|S_{N}(z)\right|: z \in K(a, R)\right\}>0 .
$$

Assume not. Then there exists a point $a \in U\left(\mu_{1}\right) \backslash V$ such that for every radius $R \in\left(0, \frac{2}{2}\right)$ we have

$$
\inf \left\{\left|S_{N}(z)\right|: z \in K(a, R)\right\}=0 \text {. }
$$

We choose a sequence of special radii $R=R_{k}$ with $R_{k} \rightarrow 0$ for $k \rightarrow \infty$. By (5.12) for each $k \in N$ there exists a point $z_{k} \in K\left(a, R_{k}\right)$ with 


$$
\left|S_{N}\left(z_{k}\right)\right|<1 / k \quad(k \in \mathbb{N})
$$

Since the measures $\mu_{1}, \ldots, \mu_{N-1}$ are discontinuous at the points of $U\left(\mu_{1}\right) \backslash V$ a short calculation shows that

$$
\left|S_{j}\left(z_{k}\right)\right| \leq \exp \left|s\left(1-\left(1 / R_{k}\right)\right)\right| \rightarrow 0 \quad(k \rightarrow \infty)
$$

for all $j \in\{1, \ldots, N-1\}$ where $s=\min _{1 \leq i \leq N-1} \mu_{i}(\{a\})$.

Now $(5.13)$ and $(5.14)$ are a contradiction to $\left(s_{1}, \ldots, S_{N}\right)=H^{\infty}$. Therefore we have established (5.11).

Since $U\left(\mu_{1}\right)$ is finite, Lemma 5.2 implies

$$
\left|S_{N}(z)\right| \geq \delta>0 \text { for all } z \in K(a)
$$

and all $a \in U\left(\mu_{1}\right) \backslash V$, where $\delta$ is some fixed positive constant. According to Lemma 5.1, (5.15) implies

$$
\sup _{a \in U\left(\mu_{1}\right) \backslash V} N\left(\mu_{N}, a\right)<\infty .
$$

Now from $(5.10)$ and $(5.16)$ we see that

$$
\sup _{a \in U\left(\mu_{1}\right)} \min _{1 \leq i \leq N} N\left(\mu_{i}, a\right)<\infty,
$$

and this implies our assertion.

\section{References}

[1] P.R. Ahern and D.N. Clark, "On inner functions with $H^{P}$-derivative", Michigan Math. J. 21 (1974), 115-127.

[2] Kenneth Hoffman, Banach spaces of conalytic functions (Prentice Hall, Englewood Cliffs, New Jersey, 1962).

[3] Walter Rudin, Real and complex analysis (McGraw-Hill, New York, London, Sydney, 1966). 
[4] Walter Rudin, "Tauberian theorems for positive harmonic functions", Nederl. Akad. Wetensch. Proc. Ser. A 81 (= Indag. Math. 40) (1978), 376-384.

[5] Stanislaw Saks, Theory of the integral, second revised edition (translated by L.C. Young. Monografie Matematyczne, 7. Lwów, Warszawa; Stechert, New York; 1937).

[6] Joel H. Shapiro, "Remarks on F-spaces of analytic functions", Banach spaces of analytic functions, 107-124 (Proc. Pelczynski Conference, Kent State University, 1976. Lecture Notes in Mathematics, 604. Springer-Verlag, Berlin, Heidelberg, New York, 1977).

Mathematisches Institut der Universität, D-6300 Giessen, West Germany. 